\title{
Desempenho agronômico de genótipos de amendoim nas condições Sorriso-MT
}

Submetido - 28 jul. $2020 \quad$ Aprovado - 20 set. $2020 \quad$ Publicado - 14 out. 2020
dol http://dx.doi.org/10.17648/sas.v1i2.43

Thiago de Souza Rizzi (D)

Engenheiro Agrônomo, Instituto Federal de Educação, Ciência e Tecnologia de Mato Grosso Campus Sorriso, Sorriso, MT, e-mail: thiago.rizzi@srs.ifmt.edu.br.

Dácio Olibone (iD

Professor do Instituto Federal de Educação, Ciência e Tecnologia de Mato Grosso - Campus Sorriso, Sorriso, MT, e-mail: dacio.olibone@srs.ifmt.edu.br, ORCID: https://orcid.org/0000-0001-8900-1023;

Jair Heuert ${ }^{(D)}$

Programa de Melhoramento do Amendoim - Embrapa, Santo Antônio de Goiás, GO, e-mail: jair.heuert@embrapa.br.

Maxuel Fellipe Nunes Xavier iD

Discente de Agronomia do Instituto Federal de Educação, Ciência e Tecnologia de Mato Grosso Campus São Vicente - Centro de Referência de Campo Verde, Campo Verde, MT, e-mail: maxuelfellipe90@gmail.com.

Discente de Agronomia do Instituto Federal de Educação, Ciência e Tecnologia de Mato Grosso Campus Sorriso, Sorriso, MT, e-mail: gabriel.mdemoura13@gmail.com.

Taís de Moraes Falleiro Suassuna (DD

Programa de Melhoramento do Amendoim - Embrapa, Santo Antônio de Goiás, GO, e-mail: tais.suassuna@embrapa.br.

\section{RESUMO}

Nos últimos anos, Sorriso se tornou o maior produtor de grãos nacional, provocando importantes transformações econômicas, sociais e na paisagem local. O presente trabalho teve como objetivo avaliar o desempenho agronômico de genótipos de amendoim nas condições de Sorriso-MT. O experimento foi realizado na área experimental do Instituto Federal de Educação, Ciência e Tecnologia de Mato Grosso - Campus Sorriso, localizado no município de Sorriso-MT, com semeadura manual realizada no dia 18 de dezembro de 2019. Foi utilizado delineamento em blocos casualizados, com quatro repetições. Os tratamentos constaram de oito genótipos de amendoim, sendo 4 cultivares (BRS 421 OL, BRS 423 OL, BRS 425 OL e EC 98 AO) e 4 linhagens (18-1253 OL, 18-1952 OL, 18-2133 OL e 18-2136 OL). As parcelas eram compostas por duas linhas de três metros de comprimento, espaçadas em 0,90 m, com intervalo de dois metros entre parcelas e área útil para avaliação de $5,4 \mathrm{~m}^{2}$ na parcela. Os manejos cultural e fitossanitário foram realizados de acordo com as recomendações para a cultura. 0 estande médio foi de 15 plantas por metro linear. $O$ ensaio foi invertido aos 135 DAE. Foram avaliados severidade de mancha preta, massa de 100 grãos, rendimento e produtividade de vagens. Nas condições experimentais em que foi desenvolvida esta pesquisa, pode-se concluir que o genótipo BRS 421 OL obteve a maior massa de 100 grãos. Os maiores rendimentos foram obtidos nos genótipos 18-2136 OL, BRS 423 OL, BRS 425 OL, 17-1253 OL e 18-1952 OL. A severidade de mancha preta foi menor para os genótipos BRS 421 OL BRS 423 OL, BRS 425 $O L$. A produtividade de vagens não sofreu influência significativa dos diferentes genótipos de amendoim. No entanto, todos genótipos produziram acima de $4.600 \mathrm{kgha}^{-1}$. Recomenda-se demais estudos para obtenção de uma possível recomendação agronômica dos genótipos mais adequados as condições de Sorriso-MT.

Palavras-chave: Arachis hypogaea L.; Programa de Melhoramento; Produtividade; Amendoim no Mato Grosso. 


\title{
Agronomic performance of peanut genotypes under Sorriso- MT conditions
}

\begin{abstract}
In the last years, Sorriso has become the largest national grain producer, causing important economic, social and local landscape transformations. This work aimed to evaluate the agronomic performance of peanut genotypes in the conditions of Sorriso-MT. The experiment was conducted in the experimental area of the Federal Institute of Education, Science and Technology of Mato Grosso - Sorriso Campus, located in the municipality of Sorriso-MT, with manual sowing performed on December 18, 2019. It was used design in randomized blocks, with four repetitions. The treatments consisted of eight peanut genotypes, being 4 cultivars (BRS 421 OL, BRS 423 OL, BRS $425 O L$ and EC 98 AO) and 4 strains (18-1253 OL, 18-1952 OL, 18-2133 $O L$ and, 18-2136 OL). The plots were composed of two lines of three meters in length, spaced $0.90 \mathrm{~m}$ apart, with an interval of two meters between plots and an evaluation area of 5.4 $\mathrm{m}^{2}$ in the plot. The crop and plant health management were carried out in accordance with the recommendations for the crop. The average stand was 15 plants per linear meter. The test was reversed at 135 DAE. Severity of black spot, mass of 100 grains, yield and yield of pods were evaluated. In the experimental conditions in which this research was developed, it can be concluded that the genotype BRS 421 OL obtained the highest mass of 100 grains. The highest yields were obtained in the genotypes 18-2136 OL, BRS 423 OL, BRS 425 OL, 17-1253 OL and 18-1952 OL. The black spot severity of was lower for genotypes BRS 421 OL BRS 423 OL, BRS $425 \mathrm{OL}$. Pod productivity was not influenced significantly influenced by the different peanut genotypes. However, all genotypes produced over 4,600 kg.ha-1. Further studies are recommended to obtain a possible agronomic recommendation of the genotypes most appropriate to the conditions of Sorriso-MT.
\end{abstract}

Keywords: Arachis hypogaea L.; Improvement Program; Productivity; Peanuts in Mato Grosso.

\section{Rendimiento agronómico de los genotipos de maní en condiciones Sorriso-MT}

\section{RESUMEN}

En los últimos años, Sorriso se ha convertido en el mayor productor nacional de granos, causando importantes cambios económicos, sociales y locales en el paisaje. El presente estudio tuvo como objetivo evaluar el rendimiento agronómico de los genotipos de maní en las condiciones de Sorriso-MT. El experimento se llevó a cabo en el área experimental del Instituto Federal de Educación, Ciencia y Tecnología de Mato Grosso - Campus Sorriso, ubicado en la ciudad de Sorriso-MT, con siembra manual realizada el 18 de diciembre de 2019. Se utilizó un diseño de bloques al azar, con cuatro repeticiones Los tratamientos consistieron en ocho genotipos de maní, 4 cultivares (BRS 421 OL, BRS 423 OL, BRS 425 OL y EC 98 AO) y 4 cepas (18-1253 OL, 18-1952 OL, 18-2133 OL y 18-2136 OL). Las parcelas consistieron en dos líneas de tres metros de longitud, espaciadas a $0.90 \mathrm{~m}$, con un intervalo de dos metros entre parcelas y un área útil para la evaluación de $5.4 \mathrm{~m}^{2}$ en la parcela. La gestión cultural y fitosanitaria se realizó de acuerdo con las recomendaciones para la cultura. El stand promedio fue de 15 plantas por metro lineal. La prueba se invirtió a 135 DAE. Se evaluaron la gravedad de la mancha negra, la masa de 100 granos, el rendimiento y el rendimiento de las vainas. En las condiciones experimentales en las que se desarrolló esta investigación, se puede concluir que el genotipo OL BRS 421 obtuvo la mayor masa de 100 granos. Los mayores rendimientos se obtuvieron para los genotipos 18-2136 OL, BRS 423 OL, BRS 425 OL, 17-1253 OL y 18-1952 OL. La severidad de la mancha negra fue menor para los genotipos BRS 421 OL BRS 423 OL, BRS $425 O L$. La productividad de la vaina no fue influenciada significativamente por los diferentes genotipos de maní. Sin embargo, todos los genotipos produjeron más de 4,600 kg.ha-1. Se recomiendan más estudios para obtener una posible recomendación agronómica de los genotipos más adecuados para las condiciones de Sorriso-MT. 
Palabras clave: Arachis hypogaea L.; Programa de mejoramiento; Productividad; Maní en Mato Grosso.

\section{Introdução}

O Brasil está ocupando a $14^{a}$ posição no ranking mundial de países produtores (USDA, 2020) sendo que já é o $5^{\circ}$ maior exportador, numa crescente demanda global por amendoim (Arachis hypogaea L.), que proporciona um crescimento sólido da cultura no Brasil. (MASSAFERA, 2020). Os produtores da Argentina, reconhecida como referência mundial na produção de amendoim, descrevem o Brasil como um gigante adormecido, por possuir um enorme potencial, dando ênfase as áreas disponíveis para expansão, principalmente no estado do Mato Grosso (TODO MANÍ, 2018).

O município de Sorriso-MT possui o título de Capital Nacional do Agronegócio. O município se consolidou no ano de 2019 como o maior exportador de Mato Grosso, representando $12,2 \%$ de todo o comércio exterior do estado e considerado $21^{\text {o }}$ no ranking nacional. As vendas externas de Sorriso podem ser resumidas em soja, com $60 \%$ da participação, milho com $39 \%$ e os $1 \%$ fica com algodão e carne (STAMM, 2019). A região é favorecida pela logística de novos modais de escoamento, como o término da pavimentação da BR-163 (FREITAS, 2020). Futuramente a Ferrogrão, uma ferrovia que consolidará um novo corredor, ligando o norte de Mato Grosso até o Porto Paraense. Canais de escoamentos esses, poderão ser utilizados para escoar a produção de amendoim local e/ou regional (MURBA, 2020)

$\mathrm{Na}$ busca da diversificação dos sistemas de produção, na safra 2018/19, iniciou um trabalho de pesquisa com amendoim em parceria entre o Instituto Federal de Mato Grosso (IFMT), Embrapa, LC Sementes e MIAC. Os resultados experimentais, com produtividades na média de $5.553 \mathrm{~kg}^{\mathrm{ha}}{ }^{-1}$ tiveram uma grande repercussão. Para o mesmo período a CONAB estimou a produtividade média no Brasil de $3.028 \mathrm{~kg} \cdot \mathrm{ha}^{-1}$. 
Esta repercussão positiva da cultura, proporcionou várias visitas em Sorriso de players brasileiros e argentinos. Como a empresa brasileira, Beatrice Comércio Importação e Exportação de Amendoim Ltda. (Beatrice Peanuts) é uma das líderes na exportação de amendoim no Brasil, tendo 42 países como destino da produção, deste montante $60 \%$ vai para exigente mercado europeu. As suas áreas de produção ficam localizadas na região de Tupã-SP com oito mil de área própria e recebe a produção de mais 15 mil oriundas de parceiros (LAZAROTTO, 2020; BAIRROS, 2020).

O município de Sorriso, pode ser região de expansão da produção de amendoim no Centro-Oeste, com grande aptidão agrícola, terras planas que facilitam a mecanização, sendo o amendoim uma alternativa promissora de diversificação dos sistemas de produção de culturas anuais. $\mathrm{O}$ amendoim encontrará boas condições edafoclimáticas, com chuvas regulares, topografia plana (favorecendo a mecanização e praticidade operacional) (FERREIRA, 2020).

Está região possui grande quantidade de áreas arenosas, onde o amendoim possui melhor desenvolvimento e rentabilidade que a soja. Logo, pode ser recomendado para cultivo em áreas adjacentes de solos mais fracos, de textura arenosas e suscetíveis a erosão (BOLONHEZI, 2020). A inserção desta nova cultura, pode ser uma opção para pequenas e médias propriedades. O presente trabalho teve como objetivo avaliar o desempenho agronômico de genótipos de amendoim nas condições de Sorriso-MT.

\section{Material e métodos}

O experimento foi realizado na área experimental do Instituto Federal de Educação, Ciência e Tecnologia de Mato Grosso - Campus Sorriso, localizada à latitude $12^{\circ} 41^{\prime} 43^{\prime \prime} \mathrm{S}$, longitude $55^{\circ} 48^{\prime} 07^{\prime \prime} \mathrm{W}$ e altitude média de $358 \mathrm{~m}$, em solo classificado como Latossolo Vermelho Amarelo distrófico com textura argilosa. O clima da região é tipo Aw, com temperatura média de $26,2^{\circ} \mathrm{C}$ e pluviosidade média de $1970 \mathrm{~mm}$ anuais os quais são distribuídos nos meses de outubro a abril (SOUZA et al., 2013). A semeadura foi manualmente, no dia 18 de dezembro de 2019. 
Foi utilizado delineamento em blocos casualizados, com quatro repetições. Os tratamentos constaram de oito genótipos de amendoim, sendo 4 cultivares (BRS 421 OL, BRS 423 OL, BRS 425 OL desenvolvidas pelo Programa de Melhoramento do Amendoim da Embrapa e a cultivar EC 98 AO desenvolvida pelo Criadero El Carmen da Argentina) e 4 linhagens (18-1253 OL, 18-1952 OL, 18-2133 OL, 18-2136 OL, desenvolvidas pelo Programa de Melhoramento do Amendoim da Embrapa).

As parcelas eram compostas por duas linhas de três metros de comprimento, espaçadas em $0,90 \mathrm{~m}$, com intervalo de dois metros entre parcelas e área útil para avaliação de $5,4 \mathrm{~m}^{2}$ na parcela. Os manejos cultural e fitossanitário foram realizados de acordo com as recomendações para a cultura. $O$ estande médio foi de 15 plantas por metro linear. $O$ ensaio foi invertido no dia 06 de maio de 2020, aos 135 dias após a emergência (DAE).

Anterior a implantação do experimento, foi efetuado o levantamento da fertilidade e granulometria do solo, utilizando-se metodologia proposta por Raij et al. (2001) e Embrapa (1997), na profundidade de 0 a 0,20 m. Os resultados das análises foram: $\mathrm{pH}\left(\mathrm{CaCl}_{2}\right)$ : 5,30; P: (resina) 5,0 mg/dm ${ }^{3} ; \mathrm{K}$ : $78 \mathrm{mg} / \mathrm{dm}^{3}$; Zn: $1,0 \mathrm{mg} / \mathrm{dm}^{3}$; Fe: $58 \mathrm{mg} / \mathrm{dm}^{3} ; \mathrm{Mn}: 0,6 \mathrm{mg} / \mathrm{dm}^{3}$; Cu: 0,3 mg/dm ${ }^{3}$; Ca: 19 mmolc/dm ${ }^{3}$; Mg: 14 mmolc/dm ${ }^{3}$; Al: 1 mmolc/dm ${ }^{3}$ e H+Al: 40 mmolc/dm ${ }^{3}$ com saturação por bases $46 \%$ e matéria orgânica $2,0 \%$. A análise granulométrica de areia, silte e argila apresentou 434, 26 e $540 \mathrm{~g} \mathrm{dm}$ 3 , respectivamente. Visando aumentar a saturação por bases para $60 \%$, foi realizado calagem de 1,4 ton ha-1 de calcário dolomítico, 60 dias antes da semeadura da primeira época, sendo este incorporado com 2 gradagens.

Foi realizada uma adubação em cobertura concomitante a plantio de $400 \mathrm{~kg} \mathrm{ha}^{-1}$ da formulação NPK 04-30-10. Aos 35 dias após a emergência foi realizada uma aplicação de gesso agrícola na dose de $500 \mathrm{~kg} \mathrm{ha}^{-1}$ a lanço na parcela. Durante o período experimental a precipitação acumulada na área foi de $894,2 \mathrm{~mm}$.

Foram avaliados severidade de mancha preta (notas) massa de 100 grãos (g), rendimento (\%) e produtividade de vagens $\left(\mathrm{kg} \mathrm{ha}^{-1} \mathrm{e}\right.$ 
sacas.alqueire $\left.{ }^{-1}\right)$. A avaliação rendimento (\%) consistiu na retirada de $100 \mathrm{~g}$ de amendoim em vagens de cada parcela sendo considerado 100\%. Após o beneficiamento mecanizado simulando um sistema industrial foi feito a massa de grãos e a casca foi desprezada, com isso calculando a porcentagem de rendimento das parcelas. A severidade de mancha preta (Cercosporidium personatum) foi avaliada usando a escala diagramática da incidência com notas de 1 a 9 antes da inversão do experimento (SUBRAHMANYAM et al., 1982). Os dados foram submetidos à análise de variância pelo Teste $\mathrm{F}$ e as médias dos tratamentos foram comparados pelo teste de Scott-Knott, no nível de 5\% de probabilidade, por meio do programa computacional SISVAR 5.6 (FERREIRA, 2019).

\section{Resultados e discussão}

Os genótipos de amendoim apresentaram diferença significativa apenas para massa de 100 grãos (g) e rendimento (\%) em função dos diferentes genótipos de amendoim. Observa-se que a severidade de mancha preta (notas) e a produtividade de vagens $\left(\mathrm{kg} \cdot \mathrm{ha}^{-1}\right)$ não foram influenciadas pelos diferentes genótipos de amendoim (Tabela 1).

Tabela 1. Severidade de mancha preta (notas), massa de 100 grãos (g), renda (\%) e produtividade de vagens $\left(\mathrm{kg} \cdot \mathrm{ha}^{-1} \mathrm{e}^{-}\right.$sacas.alqueire ${ }^{-1}$ ) em função de diferentes genótipos de amendoim. Sorriso-MT, 2019/20.

\begin{tabular}{|c|c|c|c|c|c|}
\hline \multirow{2}{*}{ Genótipos } & \multirow{2}{*}{$\begin{array}{c}\text { Severidade de } \\
\text { mancha preta } \\
\text { (notas) }\end{array}$} & \multirow{2}{*}{$\begin{array}{c}\text { Massa de } \\
100 \text { grãos } \\
(\mathrm{g})\end{array}$} & \multirow{2}{*}{$\begin{array}{c}\text { Rendimento } \\
(\%)\end{array}$} & \multicolumn{2}{|c|}{ Produtividade de vagens } \\
\hline & & & & $\left(\mathrm{kg} \cdot \mathrm{ha}^{-1}\right)$ & $\left(\right.$ sacas.alqueire $\left.^{-1}\right)$ \\
\hline BRS $421 \mathrm{OL}$ & $5,9 \mathrm{~b}$ & $76,3 \mathrm{a}$ & $77,5 \mathrm{~b}$ & $5.434,0$ & 526,0 \\
\hline BRS $425 \mathrm{OL}$ & $5,4 \mathrm{~b}$ & $69,6 \mathrm{~b}$ & $79,2 \mathrm{a}$ & $5.353,9$ & 518,3 \\
\hline BRS 423 OL & $5,7 \mathrm{~b}$ & $67,1 \mathrm{c}$ & $79,6 \mathrm{a}$ & $5.007,8$ & 484,8 \\
\hline 17-1253 OL & $6,5 \mathrm{a}$ & $68,1 \mathrm{c}$ & $78,7 \mathrm{a}$ & $4.996,3$ & 483,6 \\
\hline EC $98 \mathrm{AO}$ & $7,2 \mathrm{a}$ & $71,2 b$ & $78,1 b$ & $4.871,5$ & 471,6 \\
\hline 18-1952 OL & $7,0 \mathrm{a}$ & 69,3 b & $78,7 \mathrm{a}$ & $4.765,1$ & 461,3 \\
\hline 18-2133 OL & $6,5 \mathrm{a}$ & $63,3 \mathrm{c}$ & $76,8 b$ & $4.716,8$ & 456,6 \\
\hline 18-2136 OL & $6,7 \mathrm{a}$ & $64,3 \mathrm{c}$ & 79,8 a & $4.654,3$ & 450,5 \\
\hline C.V. (\%) & 7,96 & 5,3 & 1,3 & 7,9 & - \\
\hline $\operatorname{Pr}>\mathrm{Fc}$ & $0,0004^{*}$ & $0,0012^{*}$ & $0,0041^{*}$ & $0,0738^{\text {ns }}$ & - \\
\hline
\end{tabular}


A severidade de mancha preta, diferiu estatisticamente, sendo as menores severidades verificadas para as cultivares BRS 421 OL, BRS 423 OL e BRS $425 \mathrm{OL}$, a maior nota observada foi para a cultivar EC 98 AO, 7,2. Martins et al. (2019), obtiveram notas semelhantes com as cultivares BRS 421 OL $(5,4)$ e EC 98 AO $(7,8)$, em Santo Antônio de Goiás-GO. Ribeiro et al. (2019), também observaram notas correlatas com a cultivar BRS $421 \mathrm{OL}$ $(6,1)$ e a linhagem 18-1952 OL $(7,3)$, em Tupã-SP.

Na massa de 100 grãos (g), o genótipo BRS 421 OL (76,3 g) obteve a maior massa, quando comparado aos demais. Massas intermediárias foram obtidas nos genótipos EC 98 AO (71,2 g), BRS 425 OL (69,6 g) e 181952 OL (69,3 g). Os genótipos 17-1253 OL (68,1 g), BRS 423 OL (67,1 g), 18-2136 OL (64,3 g) e 18-2133 OL (63,3 g) obtiveram as menores massas. Corroborando com Santin et al. (2019), que obtiveram massas de 100 grãos semelhantes, com as cultivares BRS $425 \mathrm{OL}(68,0 \mathrm{~g})$ e BRS $423 \mathrm{OL}(67,5$ g), em Campo Verde-MT.

Os maiores rendimentos foram obtidos nos genótipos 18-2136 OL (79,8\%), BRS 423 OL (79,6\%), BRS 425 OL (79,2\%), 17-1253 OL (78,7\%) e 18-1952 OL (78,7\%), demonstrando que estes genótipos possuem a casca da vagem mais fina, quando comparado aos genótipos EC 98 AO (78,1\%), BRS 421 OL $(77,5 \%)$ e $18-2133$ OL $(76,8 \%)$.

A produtividade de vagens não apresentou diferença significativa em função dos diferentes genótipos de amendoim. No entanto, as cultivares BRS $421 \mathrm{OL}\left(5.434,0 \mathrm{~kg} \cdot \mathrm{ha}^{-1}\right)$, BRS $425 \mathrm{OL}\left(5.353,9 \mathrm{~kg}^{-h^{-1}}\right.$ ) e BRS $423 \mathrm{OL}$ $\left(5.007,8 \mathrm{~kg}^{\mathrm{ha}} \mathrm{h}^{-1}\right)$ foram as três mais produtivas, numericamente, produzindo acima de $5.000 \mathrm{~kg}^{-h a^{-1}}$ ou 484 sacas.alqueire $^{-1}$. A produtividade obtida na linhagem 18-2136 OL (4.654,3 kg.ha-1) é aproximadamente $24 \%$ superior a estimativa média nacional da CONAB (2020) de cultivo de amendoim primeira safra $\left(3.554,0 \mathrm{~kg} \cdot \mathrm{ha}^{-1}\right)$.

Corroborando com Rizzi et al. (2019), que também não obtiveram diferença significativa na produtividade de vagens das cultivares BRS 421 OL, BRS 423 OL, BRS 425 OL e EC 98 AO, em Sorriso-MT. Santin et al. 
(2019) também não observaram diferença significativa na produtividade das cultivares BRS 421 OL, BRS 423 OL e BRS 425 OL, em Campo Verde-MT.

\section{Conclusões}

Nas condições experimentais em que foi desenvolvida esta pesquisa, pode-se concluir que o genótipo BRS $421 \mathrm{OL}$ obteve a maior massa de 100 grãos. Os maiores rendimentos foram obtidos nos genótipos 18-2136 OL, BRS 423 OL, BRS 425 OL, 17-1253 OL e 18-1952 OL. A severidade de mancha preta foi menor para os genótipos BRS 421 OL BRS 423 OL, BRS 425 OL. A produtividade de vagens não sofreu influência significativa dos diferentes genótipos de amendoim. No entanto, todos genótipos produziram acima de $4.600 \mathrm{~kg} \cdot \mathrm{ha}^{-1}$. Recomenda-se demais estudos para obtenção de uma possível recomendação agronômica dos genótipos mais adequados as condições de Sorriso-MT.

\section{Agradecimentos}

Os autores agradecem aos discentes do curso de Engenharia Agronômica do IFMT Campus Sorriso membros do grupo de pesquisa Sistemas de Produção Agrícola no Cerrado que contribuíram na condução do experimento. A parceria institucional com o Programa de Melhoramento do Amendoim - Embrapa (vinculado ao projeto SEG 20.18.01.021.00), LC Sementes, MIAC - Indústrias Colombo e a Beatrice Peanuts. 


\section{Referências}

BAIRROS, T. Argentinos conhecem Mato Grosso para investir em amendoim. 2020. Disponível em: <http://www.sedec.mt.gov.br/-/13784741argentinos-conhecem-mato-grosso-para-investir-em-amendoim>. Acesso em: 27 jul. 2020.

BOLONHEZI, D. Cada vez mais a soja ocupa as áreas de renovação de canaviais. 2020. Disponível em: $<$ http://www.canaonline.com.br/conteudo/cada-vez-mais-a-soja-ocupa-asareas-de-renovacao-de-canaviais.html>. Acesso em: 26 jul. 2020.

CONAB. Acompanhamento da safra brasileira de grãos. Monitoramento agrícola, v. 7 - safra 2019/20 - Décimo levantamento, p. 1-74, jul. 2020. Disponível em: <https://www.conab.gov.br/info-agro/safras/graos>. Acesso em: 27 jul. 2020.

EMBRAPA. Centro Nacional de Pesquisa de Solos (Rio de Janeiro, RJ). Manual de métodos de análise de solo. 2.ed. Rio de Janeiro EmbrapaCNPS, 1997. 212p.

FERREIRA, M. Cultivo de amendoim em fazenda de Nova Ubiratã é destaque no MT Rural. 2020. Disponível em: $<$ https://www.novaubirata.mt.gov.br/imprensa/noticias/1462/>. Acesso em: 27 jul. 2020.

FERREIRA, D. F. SISVAR: um sistema de análise de computador para efeitos fixos projetos de tipo de partida dividida. Revista Brasileira de Biometria, v. 37, n. 4, p. 529-535, 2019.

FREITAS, T. Custos de frete de soja caem com pavimentação da BR163. 2020. Disponível em: <https://www.bloomberg.com.br/blog/custos-defrete-da-soja-caem-com-pavimentacao-da-br-163/>. Acesso em: 28 jul. 2020.

LAZAROTTO, C. Referência no cultivo e beneficiamento do amendoim, empresários paulistas estão de olho em Sorriso. 2020. Disponível em: $<$ https://site.sorriso.mt.gov.br/noticia/referencia-no-cultivo-e-beneficiamentodo-amendoim-empresarios-paulistas-estao-de-olho-em-sorriso5e6248f3d321d>. Acesso em: 27 jul. 2020.

MARTINS, K. B. B.; RODRIGUES, L. L.; HEUERT, J.; XAVIER, M. F. N.; SUASSUNA, T. M. F.; BETIOL, R. A. B. Desempenho agronômico de novas linhagens de amendoim no Cerrado. In: Anais do encontro sobre a cultura do amendoim, 16., 2019, Jaboticabal. Anais eletrônicos... Campinas, GALOÁ, 2019. Disponível em: <https://proceedings.science/encontro-amendoim2019/papers/desempenho-agronomico-de-novas-linhagens-de-amendoimno-cerrado>. Acesso em: 27 jul. 2020.

MASSAFERA, R. Amendoim brasileiro pode ter safra recorde este ano. 2020. Disponível em: <https://www.biodieselbr.com/noticias/materia- 
prima/outras/amendoim-brasileiro-pode-ter-safra-recorde-este-ano-160320>. Acesso em: 27 jul. 2020.

MURBA, D. Governo avança e envia ao TCU projeto da ferrovia Ferrogrão de Sinop e Miritituba. 2020. Disponível em: <https://www.sonoticias.com.br/economia/ministerio-aguarda-aprovacaopara-licitacao-da-ferrograo-de-sinop-a-miritituba/>. Acesso em: 28 jul. 2020.

RAIJ, B.; ANDRADE, J. C.; CANTARELLA, H.; QUAGGIO, J. A. Análise química para avaliação da fertilidade de solos tropicais. Campinas Instituto Agronômico, 2001. 285p.

RIBEIRO, R. P.; HEUERT, J.; SOAVE, J. H.; SANTOS, L. C. C.; SUASSUNA, T. M. F. Avaliação de severidade de mancha preta e mancha anelar em diferentes genótipos de amendoim na região da Alta Paulista. In: Anais do encontro sobre a cultura do amendoim, 16., 2019, Jaboticabal. Anais eletrônicos... Campinas, GALOÁ, 2019. Disponível em: $<$ https://proceedings.science/encontro-amendoim-2019/papers/avaliacao-deseveridade-de-mancha-preta-e-mancha-anelar-em-diferentes-genotipos-deamendoim-na-regiao-da-alta-paulista>. Acesso em: 27 jul. 2020.

RIZZI, T. S.; OLIBONE, D.; LODEA, L.; HEUERT, J.; XAVIER, M. F. N.; SUASSUNA, T. M. F. Desempenho de cultivares de amendoim na região Médio-Norte Mato-Grossense. In: Anais do encontro sobre a cultura do amendoim, 16., 2019, Jaboticabal. Anais eletrônicos... Campinas, GALOÁ, 2019. Disponível em: <https://proceedings.science/encontro-amendoim2019/papers/desempenho-de-cultivares-de-amendoim-na-regiao-medionorte-mato-grossense>. Acesso em: 27 jul. 2020.

SANTIN, V.; PEROZINI, A. C.; ARAÚJO, C.; GIRON, F. G.; HEUERT, J.; XAVIER, M. F. N.; SUASSUNA, T. M. F. Desempenho de cultivares de amendoim nas condições de Campo Verde-MT. In: Anais do encontro sobre a cultura do amendoim, 16., 2019, Jaboticabal. Anais eletrônicos... Campinas, GALOÁ, 2019. Disponível em: $<$ https://proceedings.science/encontro-amendoim-2019/papers/desempenhode-cultivares-de-amendoim-nas-condicoes-de-campo-verde-mt>. Acesso em: 27 jul. 2020.

SOUZA, A. P.; MOTA, L. L.; ZAMADEI, T. MARTIM, C. C.; ALMEIDA, F. T.; PAULINO, J. Classificação climática e balanço hídrico climatológico no estado de Mato Grosso. Nativa, v. 1, n. 1, p. 34-43, 2013.

$\begin{array}{lll}\text { STAMM, M. Valor de exportação de Sorriso aumenta } & 85 \% \text { no período de } \\ \text { janeiro a novembro. } & 2019 \text {. Disponível em: }\end{array}$ <https://www.sonoticias.com.br/economia/valor-de-exportacao-de-sorrisoaumenta-85-no-periodo-de-janeiro-a-novembro/>. Acesso em: 27 jul. 2020.

SUBRAHMANYAM, P.; MCDONALD, D.; GIBBONS, R. W.; NIGAM, S. N.; NEVILL, D. J. Resistance to rust and late leaf spot diseases in some genotypes of Arachis hypogaea. Peanut Science, v. 9, p. 9-14, 1982. 
TODO MANÍ. Um gigante adormecido. 2018. Disponível em: <http://www.todomani.com.ar/mani/notas.asp?nid=1046 >. Acesso em: 27 jul. 2020.

USDA. World Agricultural Producion. Foreign Agricultural Service, wap 520, p. 32, jul. 2020. 